\title{
Palmaris brevis spasm syndrome
}

\author{
Georges Serratrice, Jean-Philippe Azulay, Jacques Serratrice, Jean Pouget
}

\begin{abstract}
Palmaris brevis spasm syndrome is a rare and benign condition of localised muscular hyperactivity. In five men, the hypothenar eminence underwent spontaneous, irregular, tonic contractions of the palmaris brevis muscle. An EMG showed spontaneous high frequency discharges of normal motor units, without evidence of neuropathy or of nerve compression. This syndrome resembles other restricted muscle hyperactivity syndromes although there are some differences. Curiously, the palmaris brevis muscle is not under voluntary control. The mechanism of the syndrome could be an ephaptic transmission possibly secondary to the transient and repeated stretching of the ulnar nerve superficial branch. In one patient a root compression was the probable origin.
\end{abstract}

(F Neurol Neurosurg Psychiatry 1995;59:182-184)

Keywords: palmaris brevis spasm; continuous muscle hyperactivity; myokimia

Palmaris brevis spasm is a rare muscular hyperactivity syndrome ${ }^{12}$ confined to a rudimentary muscle. The mechanism is unclear. Five patients have been seen in different conditions.

\section{Case reports}

PATIENT 1

Immediately after surgery (for coxarthrosis, performed four months previously) a 76 year old man experienced paraesthesiae and hypoaesthesia in the left little finger and in the internal side of the fourth finger. During surgery, the patient had been lying on the right side and his left arm was fixed in abduction by a brace while his hand was dropping loose. Since the operation the patient had been complaining of spontaneous spasms in the left hypothenar eminence. Contractions were irregular without stereotyped duration or frequency. They occurred more often on abduction of the left abductor digiti minimi muscle. Results of a neurological examination were normal. Radiographs of the wrists were normal. An EMG showed bursts of spontaneous discharges during two to three seconds in the palmaris brevis and abductor digiti minimi muscles. The other muscles of the hand were normal. Motor unit potentials had a normal configuration with $1 \mathrm{mV}$ amplitude, recurrent with 20 to $30 \mathrm{~Hz}$ frequency. An EMG was normal with voluntary contraction. Motor and sensory nerve velocities and ulnar $F$ wave latency were normal. Carbamazepine was ineffective. Infiltration of the ulnar nerve at the wrist with lidocaine reduced the discharges but they were not abolished. Finally, there was a dramatic improvement with phenytoin ( $200 \mathrm{mg} /$ day).

\section{PATIENT 2}

A 37 year old man complained of diffuse myalgia and exercise intolerance. For eight months he had experienced difficulty in writing and had spontaneous, slightly painful, contractions in the right hypothenar eminence. Spasms (fig 1) were spontaneous and irregular. Neurological examination was normal and showed no weakness or amyotrophy. There was a diminished vibratory sensation in the feet. An EMG showed irregular discharges of high frequency. Isolated potentials were normal in amplitude $(0.5$ to $1.5 \mathrm{mV})$, configuration, and duration, in the right palmaris brevis and right abductor digiti minimi and all the other intrinsic hand muscles were normal. Motor and sensory nerve conduction velocities and $F$ wave latencies were normal. Results of routine laboratory tests including CSF and lactic acid showed no abnormalities. Magnetic resonance spectroscopy showed a delayed phosphocreatine recovery. Quadriceps muscle biopsy indicated a moderate type II fibre atrophy without any specific morphological changes. Baclofen was ineffective.

\section{PATIENT 3}

A 62 year old man had experienced a two month history of right $\mathrm{C} 8$ root pain and paraesthesiae radiating to the right little finger. He had had a trauma of the neck when aged 40 . There were spontaneous contractions and a dimple in the right hypothenar eminence. There was a weakness of the long extensor and flexor muscles of the fingers and a dorsal interossei amyotrophy. The stretching reflex of the right triceps muscle was absent. An EMG showed high amplitude $(8 \mathrm{mV})$ and frequency potentials in the right abductor digiti minimi muscle as well as in 


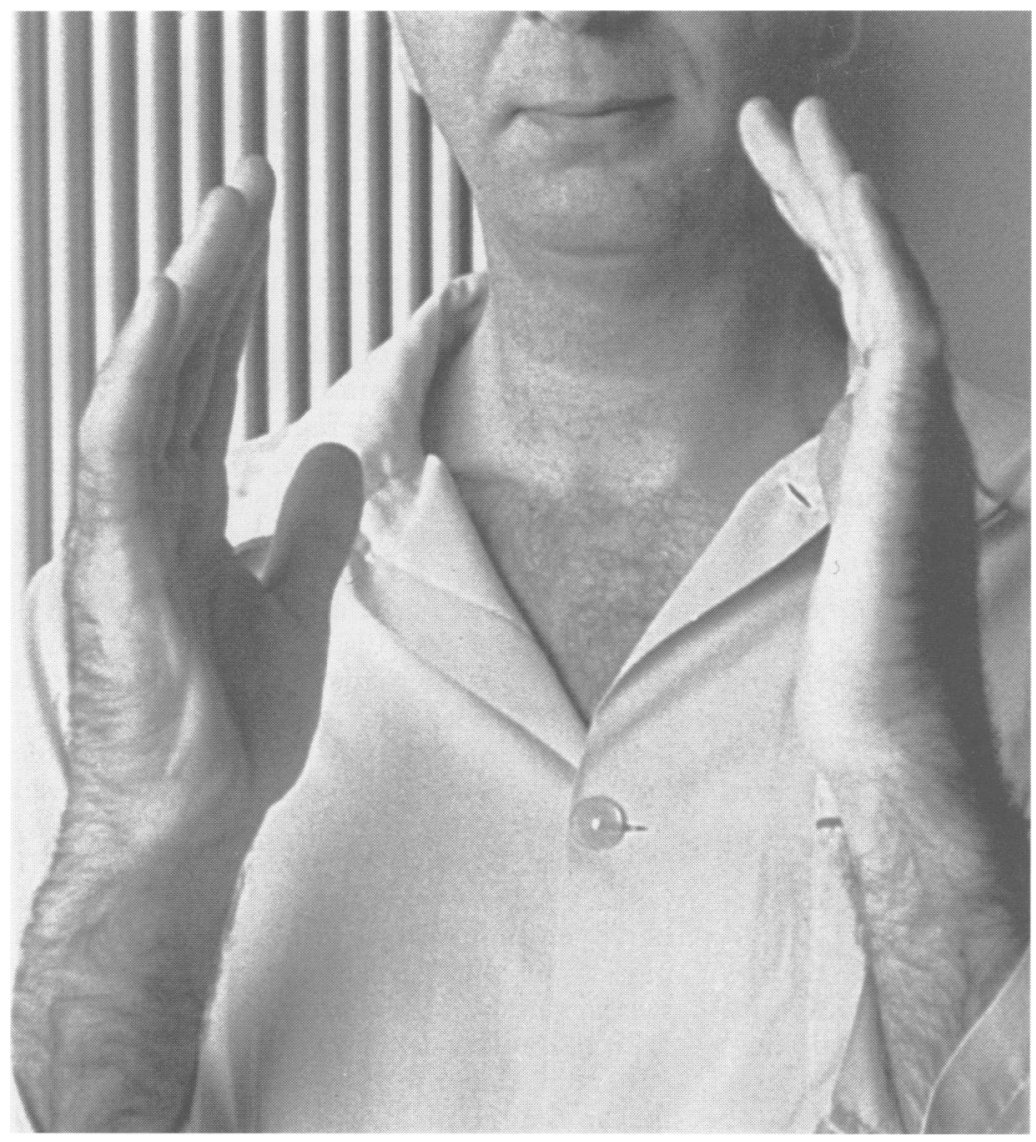

Figure 1 Patient 2; right hypothenar eminence dimple.

the finger extensors. The muscles of the arm were normal. Ulnar nerve motor conduction velocity was $65 \mathrm{~m} / \mathrm{s}$ and sensory conduction velocity was $42 \mathrm{~m} / \mathrm{s}$. At the elbow, motor conduction velocity was $36 \mathrm{~m} / \mathrm{s}$. Radiographs showed cervical osteoarthritis and a normal

Figure 2 Patient 4; contractions occurring on abduction. elbow. A cervical myelogram showed right C8 compression.

\section{PATIENT 4}

A 54 year old man complained of a two year history of paraesthesiae in both little fingers and in both fourth fingers. A year before he had had decompressive surgery of the left ulnar nerve at the elbow without any improvement. He experienced paraesthesiae and spontaneous spasms in both hypothenar eminences. Contractions occurred more often on abduction of the abductor digiti minimi (fig 2). There was hypoaesthesia in both little fingers and in the internal side of both fourth fingers. An EMG showed high amplitude and high frequency potentials in the right (14 $\mathrm{mV})$ and left $(16 \mathrm{mV})$ abductor digiti minimi muscles. Motor nerve conduction velocities and $F$ wave latencies were normal. The amplitude of sensory potentials was slightly decreased in the ulnar nerves. He was treated with phenytoin (200 mg/day) without improvement.

\section{PATIENT 5}

A 40 year old man complained of a six month history of spontaneous and irregular contraction in the ulnar side of both hands. Tonic contractions and a dimple in both hypothenar eminences were induced by a triggering gesture, when the patient put the ulnar side of his hand on a table pressing on the pisiform (sesamoid bone in the tendon of the flexor carpis ulnaris) or during wrist flexion. The spasm was aggravated after a blow on his right forearm a month before. Neurological examination was normal. An EMG showed high amplitude $(10 \mathrm{mV})$ and irregular frequency potentials in both palmaris brevis muscles. Motor and sensory nerve velocities were normal.

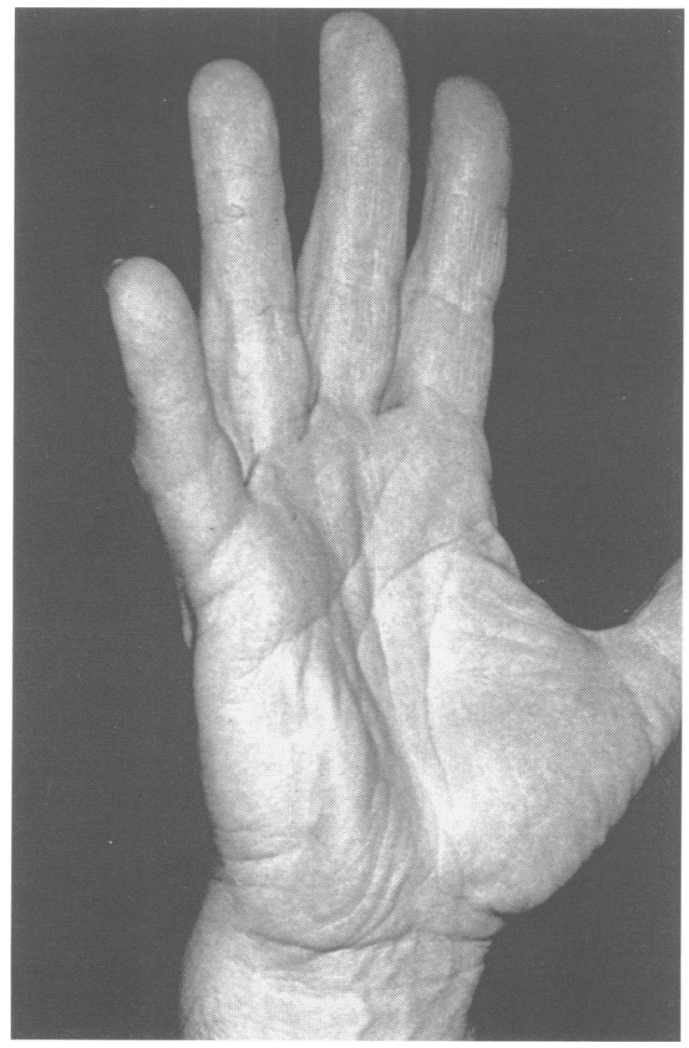

\section{Discussion}

The palmaris brevis muscle is a small, quadrilateral, thin muscle located under the skin of the hypothenar eminence. Parallel and transverse fibre bundles arise from the ulnar side of the palmaris aponeurosis. The muscle ending is located in the deep derma at the medial side of the hand. Thus the muscle action is to crease and to ripple the skin, giving an hypothenar eminence dimple. Interestingly, the palmaris brevis muscle is not under voluntary control and its contraction is strictly involuntary. The ulnar nerve divides into superficial and deep terminal branches and the superficial branch extends distally. A small twig supplies the palmaris brevis muscle. At the distal border of this muscle the superficial branch is divided into palmar digital branches for the ulnar side of the little finger and the fourth interdigital space. It is important to note that this ulnar superficial branch has both motor and sensory functions whereas the twig supplying the palmaris brevis muscle is a motor nerve only. Theoretically a nerve entrapment causes motor and sensory disturbances. 
The symptoms of palmaris brevis muscle spasm are similar in three other reported patients ${ }^{12}$ and in our five patients but there are a few differences. The main clinical characteristics are (a) frequency in older men; (b) isolated contractions of the palmaris brevis muscle; (c) spontaneous, brisk, irregular tonic contractions; $(d)$ dimpling in the ulnar side of the hand without stereotyped duration or frequency; (e) usually no triggering factors; the patient is unable to initiate the contraction at will and the contractions start and stop without any apparent cause. The voluntary contraction of the little finger (patient 4) or a pressure on the pisiform (patient 5) could sometimes trigger the dimpling; $(f)$ benign evolution for several years; $(g)$ aggravation under stress or excitement, decrease at rest or during sleep; $(h)$ spasms are either bilateral (five patients) or unilateral (three patients); (i) there is no muscle hyperactivity in the hypothenar muscles or in any other muscle of the body; (j) sensory disturbances are inconsistent.

At rest, EMG shows high frequency discharges of motor units with normal amplitude, duration, and morphology. Discharges occur spontaneously, last a short time, and recur at irregular intervals. They occur only in the palmaris brevis muscle, except in three patients (patient 2 of our series), in whom the abductor digiti minimi muscle fired occasionally for brief periods during the spasm. By contrast all other intrinsic hand or arm muscles were electrically silent during the spasm. Moreover, voluntary abduction of the little finger did not trigger any discharges except in three cases. Motor and sensory nerve conduction velocities were normal, specially in the ulnar nerve, except in patient 3 of our series in whom a double crush syndrome was probable considering the reduction of the motor nerve velocity at the elbow; $\mathrm{F}$ wave latencies were normal. There was no block after proximal motor nerve stimulation. In two patients ${ }^{2}$ lidocaine infiltrations of the ulnar nerve at the wrist resulted in complete nerve block abolishing both spasm and spontaneous discharges whereas in patient 1 of our series, discharges were only reduced by infiltrations. Carbamazepine was ineffective in our patients and baclofen was ineffective in patient 2 . On the other hand there was a dramatic improvement with phenytoin in patient 1 of our series.

The mechanism of the spasm restricted to the palmaris brevis muscle is unclear and the aetiology could be different in each of our five patients. In patient 1 , the spontaneous discharges arose in areas of hypothetical functional focal damage in a stretched peripheral nerve, possibly through ephaptic transmission. In patient 2 , the exact site of the abnormal spontaneous discharges is unknown. Abnormalities in membrane channels could create rhythmic generators on the axon. In patient 3, a root compression with a probable double crush at the elbow can be considered as a possible mechanism. In the same way myokimia of the calf associated with S1 radiculopathy is well known..$^{3-5}$ In patient 4, a nerve entrapment of the whole superficial branch of the ulnar nerve could result in hypoaesthesia in the distal territory. A nerve focal lesion can cause localised myokimia. ${ }^{6}$ In patient 5, pressure on the pisiform triggered the spasm. Thus the site of the abnormal spontaneous discharge varied.

1 Satya-Murti S, Layzer RB. Hypothenar dimpling: a peripheral equivalent of hemifacial spasm? Arch Neurol peripheral equivale

2 Loron P, Bouche P, Marolle L, Gautier JC. Le spasme ou fossette de l'éminence hypothénar. Rev Neurol (Paris) 1985;141:149-51.

3 Lapresle J, Fardeau M, Said G. L'hypertrophie musculaire vraie secondaire à une atteinte nerveuse périphérique. Rev Neurol (Paris) 1973;128:153-60.

4 Ricker K, Rohkamm R, Moxley RT III. Hypertrophy of the calf with $S_{1}$ radiculopathy. Arch Neurol 1988;45: 660-4.

5 Serratrice G, Pellissier JF, Marini JF, Valentin P. Un cas de sciatique avec hypertrophie du mollet. Rev Neurol (Paris) 1989;145:474-7.

6 Medina IL, Chokroverty S, Reyes M. Localized myokimia caused by peripheral nerve injury. Arch Neurol 1976; 33:587-8. 